\title{
Applications of a version of the de Rham lemma to the existence theory of a weak solution to the Maxwell-Stokes type equation
}

Received: 15 February 2018 / Accepted: 19 September 2018 / Published online: 28 September 2018 (C) The Author(s) 2018

\begin{abstract}
In this paper, we show the existence of a weak solution to the Maxwell-Stokes type equation with a potential satisfying the Dirichlet condition, under the hypothesis that the domain has no holes, using a version of the de Rham lemma that was proved in our previous paper. We also give the regularity of weak solutions.

Mathematics Subject Classification $35 \mathrm{~J} 50 \cdot 35 \mathrm{~A} 15 \cdot 35 \mathrm{D} 05 \cdot 35 \mathrm{~A} 05$

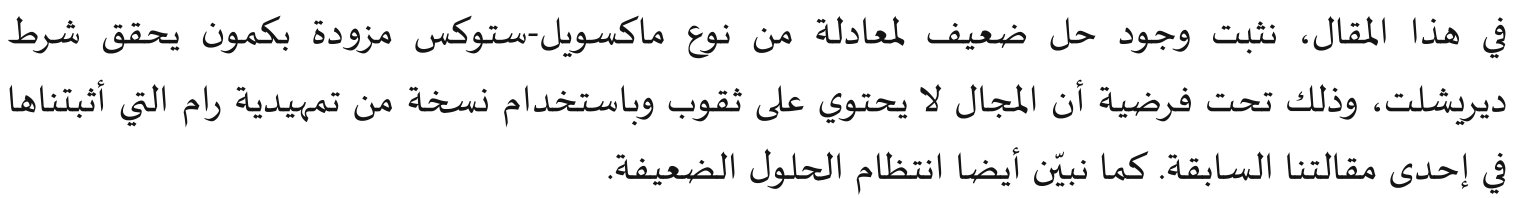

\section{Introduction}

In this paper, we consider the existence and regularity of a weak solution to a class of the Maxwell-Stokes system containing a $p$-curl system in a bounded domain $\Omega$ of $\mathbb{R}^{3}$.

If $\Omega$ is bounded, simply connected and has no holes, Yin [15] showed the existence of a unique weak solution for a so-called $p$-curl system

$$
\begin{cases}\operatorname{curl}\left[|\operatorname{curl} \boldsymbol{u}|^{p-2} \operatorname{curl} \boldsymbol{u}\right]=\boldsymbol{f} & \text { in } \Omega, \\ \operatorname{div} \boldsymbol{u}=0 & \text { in } \Omega, \\ \boldsymbol{n} \times \boldsymbol{u}=\mathbf{0} & \text { on } \Gamma,\end{cases}
$$

where $\Gamma$ is the $C^{2, \alpha}$ boundary of $\Omega, p>1, \boldsymbol{n}$ is the unit outer normal vector to $\Gamma$ and $\boldsymbol{f} \in \boldsymbol{C}^{\alpha}(\bar{\Omega})$ is a given vector field satisfying div $f=0$ in $\Omega$. Moreover, he got the optimal $C^{1, \beta}$ regularity of the weak solution for some $\beta \in(0,1)$. See also Yin [16] and Yin et al. [17]. The system (1.1) is a steady-state approximation of Bean's critical state model for type II superconductors (cf. [9,14]).

J. Aramaki $(\varangle)$

Division of Science, Faculty of Science and Engineering, Tokyo Denki University, Hatoyama-machi, Saitama 350-0394, Japan

E-mail: aramaki@hctv.ne.jp 
Aramaki [3] extended the results to a more general system:

$$
\begin{cases}\operatorname{curl}\left[S_{t}\left(|\operatorname{curl} \boldsymbol{u}|^{2}\right) \operatorname{curl} \boldsymbol{u}\right]=\boldsymbol{f} & \text { in } \Omega, \\ \operatorname{div} \boldsymbol{u}=0 & \text { in } \Omega, \\ \boldsymbol{n} \times \boldsymbol{u}=\mathbf{0} & \text { on } \Gamma=\partial \Omega,\end{cases}
$$

where $S(t) \in C^{2}((0, \infty)) \cap C^{0}([0, \infty))$ satisfies some structure conditions.

In a multi-connected bounded domain, the systems (1.1) and (1.2) are not well posed. In this case, if $\Gamma$ has a finite connected components $\Gamma_{0}, \Gamma_{1}, \ldots, \Gamma_{m}$ with $\Gamma_{0}$ denoting the boundary of the infinite connected component of $\mathbb{R}^{3} \backslash \bar{\Omega}$, Aramaki [4] considered the following system:

$$
\begin{cases}\operatorname{curl}\left[S_{t}\left(x,|\operatorname{curl} \boldsymbol{u}|^{2}\right) \operatorname{curl} \boldsymbol{u}\right]=\boldsymbol{f} & \text { in } \Omega, \\ \operatorname{div} \boldsymbol{u}=0 & \text { in } \Omega, \\ \boldsymbol{n} \times \boldsymbol{u}=\mathbf{0} & \text { on } \Gamma, \\ \langle\boldsymbol{u} \cdot \boldsymbol{n}, 1\rangle_{\Gamma_{i}}=0 & \text { for } i=1,2, \ldots, m,\end{cases}
$$

where $S(x, t) \in C^{2}(\Omega \times(0, \infty)) \cap C^{0}(\Omega \times[0, \infty))$ satisfies some structure conditions given in Sect. 2 and $\langle\cdot, \cdot\rangle_{\Gamma_{i}}$ denotes the duality between $\boldsymbol{W}^{-1 / p, p}\left(\Gamma_{i}\right)$ and $\boldsymbol{W}^{1-1 / p^{\prime}, p^{\prime}}\left(\Gamma_{i}\right)$. Here $p^{\prime}$ is the conjugate exponent of $p$, that is, $(1 / p)+\left(1 / p^{\prime}\right)=1$. If $\boldsymbol{f} \in \boldsymbol{C}^{\alpha}(\bar{\Omega})$ satisfies

$$
\operatorname{div} \boldsymbol{f}=0 \text { in } \Omega \text { and }\langle\boldsymbol{f} \cdot \boldsymbol{n}, 1\rangle_{\Gamma_{i}}=0 \quad \text { for } i=1,2, \ldots, m,
$$

then we showed that (1.3) has a unique weak solution $\boldsymbol{u}$ and the weak solution belongs to $\boldsymbol{C}^{1, \beta}(\bar{\Omega})$, and there exists a constant $C>0$ depending only on $p, \Omega$ and $\|\boldsymbol{f}\|_{C^{\alpha}(\bar{\Omega})}$ such that

$$
\|\boldsymbol{u}\|_{C^{1, \beta}(\bar{\Omega})} \leq C .
$$

If the hypothesis $\operatorname{div} f=0$ in $\Omega$ is not satisfied, we may consider the following Maxwell-Stokes type problem: to find $(\boldsymbol{u}, \pi)$ in an appropriate space such that

$$
\begin{cases}\operatorname{curl}\left[S_{t}\left(x,|\operatorname{curl} \boldsymbol{u}|^{2}\right) \operatorname{curl} \boldsymbol{u}\right]=\boldsymbol{f}+\nabla \pi & \text { in } \Omega, \\ \operatorname{div} \boldsymbol{u}=0 & \text { in } \Omega, \\ \boldsymbol{n} \times \boldsymbol{u}=\mathbf{0} & \text { on } \Gamma, \\ \langle\boldsymbol{u} \cdot \boldsymbol{n}, 1\rangle_{\Gamma_{i}}=0 & \text { for } i=1,2, \ldots, m\end{cases}
$$

In this situation, the conditions corresponding to (1.4) imply

$$
\begin{cases}\Delta \pi=-\operatorname{div} \boldsymbol{f} & \text { in } \Omega \\ \frac{\partial \pi}{\partial \boldsymbol{n}}=-\boldsymbol{f} \cdot \boldsymbol{n} & \text { on } \Gamma\end{cases}
$$

That is to say, $\pi$ must be a solution of the Neumann problem for the Poisson equation. This case was considered in Aramaki [6].

In this paper, we consider the case where $\Omega$ has no holes. If $\Omega$ has no holes, i.e., $m=0$, then the last condition of (1.5) is unnecessary. In this situation, we can consider the following Maxwell-Stokes problem: to find $(\boldsymbol{u}, \pi)$ in an appropriate space such that

$$
\begin{cases}\operatorname{curl}\left[S_{t}\left(x,|\operatorname{curl} \boldsymbol{u}|^{2}\right) \operatorname{curl} \boldsymbol{u}\right]=\boldsymbol{f}+\nabla \pi & \text { in } \Omega, \\ \operatorname{div} \boldsymbol{u}=0 & \text { in } \Omega, \\ \boldsymbol{n} \times \boldsymbol{u}=\mathbf{0} & \text { on } \Gamma\end{cases}
$$

with the Dirichlet boundary condition for $\pi$ instead of (1.6):

$$
\begin{cases}\Delta \pi=-\operatorname{div} f & \text { in } \Omega \\ \pi=0 & \text { on } \Gamma\end{cases}
$$

In the present paper, we consider such a case. To do so, we must apply a version of the de Rham lemma.

The classical de Rham lemma says that a continuous and linear functional that vanishes on all divergencefree $H^{1}$ vector fields that equal zero on the boundary can be represented as a gradient of an $L^{2}$ potential 
function inside the domain. For example, see Boyer and Fabrie [8, Theorem IV. 2.4]. However, the lemma does not provide any information on the trace of the potential function on the boundary.

For example, it is insufficient to show the existence of solutions for the Stokes problem. Pan [13] considered a version of the de Rham lemma. This asserts that a continuous and linear functional that vanishes on all divergence-free $H^{1}$ vector fields that have zero tangential component on the boundary is a gradient of the function $\pi \in L^{2}(\Omega)$, and that $\pi$ has zero trace on the boundary. This additional information on the trace of $\pi$ makes it possible to improve the existence of a solution of the Maxwell-Stokes type system. However, it is insufficient to consider the existence of a weak solution to the Maxwell-Stokes type problem containing a $p$-curl system with the potential satisfying the Dirichlet condition.

In our previous paper Aramaki [5], we considered the $L^{p}$ version of the result obtained by [13]. Our result is useful for the existence of a weak solution to the Maxwell-Stokes type problem in the $L^{p}$ version, .

The paper is organized as follows. In Sect. 2, we give some preliminaries on the trace and the gradient of functions. In Sect. 3, we give a main theorem on the existence of a weak solution to the Maxwell-Stokes type equation using the variational method under the hypothesis that $\Omega$ has no holes. In Sect. 4, we consider the regularity of the weak solution in the case where $\Omega$ has no holes.

\section{Preliminaries}

In this section, we state some preliminaries that are necessary later. Let $\Omega$ be a bounded domain in $\mathbb{R}^{3}$ with a $C^{2}$ boundary $\Gamma, 1<p<\infty$ and let $p^{\prime}$ be the conjugate exponent i.e., $(1 / p)+\left(1 / p^{\prime}\right)=1$. From now on we use $C^{m}(\bar{\Omega}), C^{m, \alpha}(\bar{\Omega}), L^{p}(\Omega), W^{m, p}(\Omega)(m \geq 0$, integer $), W^{s, p}(\Gamma)(s \in \mathbb{R})$, and so on, for the standard Hölder spaces, Sobolev spaces of functions. For any Banach space $B$, we denote $B \times B \times B$ by boldface character $\boldsymbol{B}$. Hereafter, we use this character to denote vector and vector-valued functions, and we denote the standard inner product of vectors $\boldsymbol{a}$ and $\boldsymbol{b}$ in $\mathbb{R}^{3}$ by $\boldsymbol{a} \cdot \boldsymbol{b}$. Moreover, for the dual space $\boldsymbol{B}^{\prime}$ of $\boldsymbol{B}$, we denote the duality between $\boldsymbol{B}^{\prime}$ and $\boldsymbol{B}$ by $\langle\cdot, \cdot\rangle_{\boldsymbol{B}^{\prime}, \boldsymbol{B}}$. If $Y(\Omega)$ is a space of functions defined in $\Omega$, we denote

$$
\dot{Y}(\Omega)=\left\{\phi \in Y(\Omega) ; \int_{\Omega} \phi \mathrm{d} x=0\right\} .
$$

Define a space

$$
\boldsymbol{W}_{t 0}^{1, p}(\Omega)=\left\{\boldsymbol{u} \in \boldsymbol{W}^{1, p}(\Omega) ; \boldsymbol{u}_{T}=\mathbf{0} \text { on } \Gamma\right\},
$$

where $\boldsymbol{u}_{T}$ denotes the tangential component of the trace of $\boldsymbol{u}$, namely, $\boldsymbol{u}_{T}=(\boldsymbol{n} \times \boldsymbol{u}) \times \boldsymbol{n}, \boldsymbol{n}$ is the outer unit normal vector to the boundary, and we denote its dual space by $\boldsymbol{W}_{t 0}^{1, p}(\Omega)^{\prime}$. Moreover, we define

$$
\boldsymbol{W}_{t 0}^{1, p}(\Omega, \operatorname{div} 0)=\left\{\boldsymbol{u} \in \boldsymbol{W}_{t 0}^{1, p}(\Omega) ; \operatorname{div} \boldsymbol{u}=0 \text { in } \Omega\right\} .
$$

We define the norm of elements of $\dot{C}^{1}(\bar{\Omega})$ by

$$
\|\phi\|_{p^{\prime},-1 / p^{\prime}}=\|\phi\|_{L^{p^{\prime}(\Omega)}}+\left\|\gamma_{0}(\phi)\right\|_{W^{-1 / p^{\prime}, p^{\prime}(\Gamma)}},
$$

where $\gamma_{0}$ is the restriction operator to the boundary $\Gamma$, and the completion of $\dot{C}^{1}(\bar{\Omega})$ with respect to this norm by $\dot{L}^{p^{\prime},-1 / p^{\prime}}(\Omega)$ and define

$$
L^{p^{\prime},-1 / p^{\prime}}(\Omega)=\dot{L}^{p^{\prime},-1 / p^{\prime}}(\Omega) \oplus \mathbb{R} .
$$

We give a proposition associated with the trace and the gradient (cf. [5]).

Proposition 2.1 Let $\Omega$ be a bounded domain in $\mathbb{R}^{3}$ with a $C^{2}$ boundary $\Gamma$. Then the following holds.

(i) There exists a trace map $\gamma: L^{p^{\prime},-1 / p^{\prime}}(\Omega) \rightarrow W^{-1 / p^{\prime}, p^{\prime}}(\Gamma)$ such that if $\phi \in \dot{C}^{1}(\bar{\Omega})$, then $\gamma(\phi)=\gamma_{0}(\phi)$. 
(ii) For given $\phi \in L^{p^{\prime},-1 / p^{\prime}}(\Omega)$, we define a bounded linear functional on $\boldsymbol{W}_{t 0}^{1, p}(\Omega)$, which is denoted by $\nabla \phi$, for all $\boldsymbol{w} \in \boldsymbol{W}_{t 0}^{1, p}(\Omega)$,

$$
\begin{aligned}
& \langle\nabla \phi, \boldsymbol{w}\rangle_{\boldsymbol{W}_{t 0}^{1, p}(\Omega)^{\prime}, \boldsymbol{W}_{t 0}^{1, p}(\Omega)} \\
& =-\int_{\Omega} \phi \operatorname{div} \boldsymbol{w} \mathrm{d} x+\langle\gamma(\phi), \boldsymbol{n} \cdot \boldsymbol{w}\rangle_{W^{-1 / p^{\prime}, p^{\prime}}(\Gamma), W^{1-1 / p, p}(\Gamma)}
\end{aligned}
$$

If we write

$$
\operatorname{grad} L^{p^{\prime},-1 / p^{\prime}}(\Omega)=\left\{\nabla \phi \in \boldsymbol{W}_{t 0}^{1, p}(\Omega)^{\prime} ; \phi \in L^{p^{\prime},-1 / p^{\prime}}(\Omega)\right\},
$$

then $\operatorname{grad} L^{p^{\prime},-1 / p^{\prime}}(\Omega)$ is a closed subspace of $\boldsymbol{W}_{t 0}^{1, p}(\Omega)^{\prime}$, and it is homeomorphic to $\dot{L}^{p^{\prime},-1 / p^{\prime}}(\Omega)$.

Now we give a $L^{p}$ version of the de Rham lemma that was proved by our previous paper [5].

Theorem 2.2 Let $\Omega$ be a bounded domain in $\mathbb{R}^{3}$ with a $C^{2}$ boundary $\Gamma$. Assume that $T \in W_{t 0}^{1, p}(\Omega)^{\prime}$ satisfies

$$
\langle T, \boldsymbol{w}\rangle_{\boldsymbol{W}_{t 0}^{1, p}(\Omega)^{\prime}, \boldsymbol{W}_{t 0}^{1, p}(\Omega)}=0 \text { for all } \boldsymbol{w} \in \boldsymbol{W}_{t 0}^{1, p}(\Omega, \operatorname{div} 0) .
$$

Then there exists $\pi \in L^{p^{\prime},-1 / p^{\prime}}(\Omega)$ such that $\gamma(\pi)=0$ on $\Gamma$ and $T=\nabla \pi$ on $\boldsymbol{W}_{t 0}^{1, p}(\Omega)^{\prime}$.

We assume that $\Omega$ has the following conditions as in Amrouche and Seloula [1] (cf. [2,10,12]). Let $\Omega \subset \mathbb{R}^{3}$ be a bounded domain of class $C^{r, \alpha}(r \geq 1)$ with the boundary $\Gamma$ and $\Omega$ be locally situated on one side of $\Gamma$.

1. $\Gamma$ has a finite number of connected components $\Gamma_{0}, \Gamma_{1}, \ldots, \Gamma_{m}$ with $\Gamma_{0}$ denoting the boundary of the infinite connected component of $\mathbb{R}^{3} \backslash \bar{\Omega}$.

2. There exist $n$ connected open surfaces $\Sigma_{j},(j=1, \ldots, n)$, called cuts, contained in $\Omega$ such that

(a) $\Sigma_{j}$ is an open subset of a smooth manifold $\mathcal{M}_{j}$.

(b) $\partial \Sigma_{j} \subset \Gamma(j=1, \ldots, n)$, where $\partial \Sigma_{j}$ denotes the boundary of $\Sigma_{j}$, and $\Sigma_{j}$ is non-tangential to $\Gamma$.

(c) $\overline{\Sigma_{i}} \cap \overline{\Sigma_{j}}=\emptyset(i \neq j)$.

(d) The open set $\dot{\Omega}=\Omega \backslash\left(\cup_{i=1}^{n} \Sigma_{i}\right)$ is simply connected and pseudo $C^{1,1}$ class.

The number $n$ is called the first Betti number which is equal to the number of handles of $\Omega$, and $m$ is called the second Betti number which is equal to the number of holes. We say that if $n=0, \Omega$ is simply connected, and if $m=0, \Omega$ has no holes.

Define two spaces by

$$
\begin{aligned}
& \mathbb{K}_{N}^{p}(\Omega)=\left\{\boldsymbol{v} \in \boldsymbol{L}^{p}(\Omega) ; \operatorname{div} \boldsymbol{v}=0, \operatorname{curl} \boldsymbol{v}=\mathbf{0} \text { in } \Omega, \boldsymbol{v} \times \boldsymbol{n}=\mathbf{0} \text { on } \Gamma\right\}, \\
& \mathbb{K}_{T}^{p}(\Omega)=\left\{\boldsymbol{v} \in \boldsymbol{L}^{p}(\Omega) ; \operatorname{div} \boldsymbol{v}=0, \operatorname{curl} \boldsymbol{v}=\mathbf{0} \text { in } \Omega, \boldsymbol{v} \cdot \boldsymbol{n}=0 \text { on } \Gamma\right\} .
\end{aligned}
$$

Then it is well known that $\operatorname{dim} \mathbb{K}_{T}^{p}(\Omega)=n$ and $\operatorname{dim} \mathbb{K}_{N}^{p}(\Omega)=m$.

We assume that a function $S(x, t) \in C^{2}(\Omega \times(0, \infty)) \cap C^{0}(\Omega \times[0, \infty))$ satisfies the following structure conditions. There exist $1<p<\infty$ and positive constants $0<\lambda<\Lambda$ such that for all $x \in \Omega$

$$
\begin{aligned}
& S(x, 0)=0 \text { and } \lambda t^{(p-2) / 2} \leq S_{t}(x, t) \leq \Lambda t^{(p-2) / 2} \text { for } t>0 \\
& \lambda t^{(p-2) / 2} \leq S_{t}(x, t)+2 t S_{t t}(x, t) \leq \Lambda t^{(p-2) / 2} \text { for } t>0 \\
& \text { If } 1<p<2, S_{t t}(x, t)<0, \text { and if } p \geq 2, S_{t t}(x, t) \geq 0 \text { for } t>0 . \\
& \left|S_{t x}(x, t)\right| \leq \Lambda t^{(p-2) / 2} \text { for } t>0 .
\end{aligned}
$$

We note that from (2.3a), we have

$$
\frac{2}{p} \lambda t^{p / 2} \leq S(x, t) \leq \frac{2}{p} \Lambda t^{p / 2} \text { for } t \geq 0
$$

For example, if $S(x, t)=v(x) t^{p / 2}$, where $v \in C^{2}(\Omega)$ satisfies $0<v_{*} \leq v(x) \leq v^{*}<\infty$ and $|\nabla v(x)| \leq C$, then it follows from elementary calculations that (2.3a)-(2.3d) hold. 


\section{Existence of a weak solution to the Maxwell-Stokes type system}

In this and next sections, we assume that $\Omega$ has no holes, that is, $m=\operatorname{dim} \mathbb{K}_{N}^{p}(\Omega)=0$.

We consider the following Maxwell-Stokes type system

$$
\begin{cases}\operatorname{curl}\left[S_{t}\left(x,|\operatorname{curl} \boldsymbol{u}|^{2}\right) \operatorname{curl} \boldsymbol{u}\right]=\boldsymbol{f}(x, \boldsymbol{u})+\nabla \pi & \text { in } \Omega, \\ \operatorname{div} \boldsymbol{u}=0 & \text { in } \Omega, \\ \boldsymbol{u}_{T}=\boldsymbol{u}_{T}^{0} & \text { on } \Gamma . \\ \pi=0 & \text { on } \Gamma .\end{cases}
$$

Here $\boldsymbol{u}_{T}^{0}$ is a given vector field on $\Gamma$ such that $\boldsymbol{n} \cdot \boldsymbol{u}_{T}^{0}=0$ on $\Gamma$. Moreover, $\boldsymbol{f}(x, \boldsymbol{z})$ is a given Carathéodory function on $\Omega \times \mathbb{R}^{3}$ satisfying that there exists a constant $M>0$ such that

$$
|\boldsymbol{f}(x, z)| \leq M\left(1+|z|^{p-1}\right) \text { for all }(x, z) \in \Omega \times \mathbb{R}^{3} .
$$

Define two function spaces

$$
\begin{aligned}
\boldsymbol{W}^{1, p}(\Omega, \operatorname{div} 0) & =\left\{\boldsymbol{v} \in \boldsymbol{W}^{1, p}(\Omega) ; \operatorname{div} \boldsymbol{v}=0 \text { in } \Omega\right\}, \\
\boldsymbol{W}_{t}^{1, p}\left(\Omega, \operatorname{div} 0, \boldsymbol{u}_{T}^{0}\right) & =\left\{\boldsymbol{v} \in \boldsymbol{W}^{1, p}(\Omega, \operatorname{div} 0) ; \boldsymbol{u}_{T}=\boldsymbol{u}_{T}^{0} \text { on } \Gamma\right\} .
\end{aligned}
$$

Definition 3.1 We say $(\boldsymbol{u}, \pi)$ is a weak solution of (3.1), if $(\boldsymbol{u}, \pi) \in \boldsymbol{W}_{t}^{1, p}\left(\Omega, \operatorname{div} 0, \boldsymbol{u}_{T}^{0}\right) \times L^{p^{\prime},-1 / p^{\prime}}(\Omega)$, and satisfies $\pi=0$ on $\Gamma$ in the sense of trace and

$$
\begin{aligned}
& \int_{\Omega} S_{t}\left(x,|\operatorname{curl} \boldsymbol{u}|^{2}\right) \operatorname{curl} \boldsymbol{u} \cdot \operatorname{curl} \boldsymbol{v} \mathrm{d} x=\int_{\Omega} \boldsymbol{f}(x, \boldsymbol{u}) \cdot \boldsymbol{v} \mathrm{d} x \\
& \quad+\langle\nabla \pi, \boldsymbol{v}\rangle_{\boldsymbol{W}_{t 0}^{1, p}(\Omega)^{\prime}, \boldsymbol{W}_{t 0}^{1, p}(\Omega)} \text { for all } \boldsymbol{v} \in \boldsymbol{W}_{t 0}^{1, p}(\Omega) .
\end{aligned}
$$

Here if $\pi \in L^{p^{\prime},-1 / p^{\prime}}(\Omega)$, then $\nabla \pi \in \boldsymbol{W}_{t 0}^{1, p}(\Omega)^{\prime}$ is well defined from Proposition 2.1(ii).

We assume that there exists a scalar function $F(x, z)$ which is measurable in $x \in \Omega$ and differentiable in $z \in \mathbb{R}^{3}$ such that

$$
\nabla_{z} F(x, z)=f(x, z), \quad|F(x, z)| \leq M\left(1+|z|^{p}\right) \text { for all }(x, z) \in \Omega \times \mathbb{R}^{3}
$$

and

$$
\lim _{|z| \rightarrow \infty} \frac{|F(x, z)|}{|z|^{p}}=0 \text { uniformly in } x \in \Omega .
$$

We are in a position to state the theorem.

Theorem 3.2 Let $\Omega$ be a bounded domain with a $C^{2}$ boundary $\Gamma$ satisfying (1) and (2) with $m=0$, that is, $\Omega$ has no holes. Assume that a given function $S(x, t)$ satisfies (2.3a)-(2.3d) and a given function $\boldsymbol{f}(x, \boldsymbol{z})$ satisfies (3.4) and (3.5). Then (3.1) has a weak solution

$$
(\boldsymbol{u}, \pi) \in \boldsymbol{W}_{t}^{1, p}\left(\Omega, \operatorname{div} 0, \boldsymbol{u}_{T}^{0}\right) \times L^{p^{\prime},-1 / p^{\prime}}(\Omega) .
$$

Furthermore, if $\boldsymbol{f}$ satisfies

$$
\left(f\left(x, z_{2}\right)-f\left(x, z_{1}\right)\right) \cdot\left(z_{2}-z_{1}\right) \leq 0 \text { for all } x \in \Omega, z_{1}, z_{2} \in \mathbb{R}^{3},
$$

then the weak solution is unique.

Proof We use the standard variational method. Define a functional on $\boldsymbol{W}_{t}^{1, p}\left(\Omega, \operatorname{div} 0, \boldsymbol{u}_{T}^{0}\right)$ by

$$
\mathcal{E}[\boldsymbol{u}]=\int_{\Omega}\left(\frac{1}{2} S\left(x,|\operatorname{curl} \boldsymbol{u}|^{2}\right)-F(x, \boldsymbol{u})\right) \mathrm{d} x
$$


and put

$$
c_{0}=\inf _{\boldsymbol{u} \in \boldsymbol{W}_{t}^{1, p}\left(\Omega, \operatorname{div} 0, \boldsymbol{u}_{T}^{0}\right)} \mathcal{E}[\boldsymbol{u}] .
$$

By (3.5), we can see that for any $\varepsilon>0$, there exists a constant $C_{\varepsilon}>0$ such that

$$
|F(x, z)| \leq \varepsilon|z|^{p}+C_{\varepsilon} \text { for all }(x, z) \in \Omega \times \mathbb{R}^{3} .
$$

By [1, Corollary 5.2], for any $\boldsymbol{v} \in \boldsymbol{W}^{1, p}(\Omega)$, we have

$$
\begin{aligned}
\|\boldsymbol{v}\|_{\boldsymbol{W}^{1, p}(\Omega)} \leq & C\left(\|\boldsymbol{v}\|_{\boldsymbol{L}^{p}(\Omega)}+\|\operatorname{curl} \boldsymbol{v}\|_{\boldsymbol{L}^{p}(\Omega)}\right. \\
& \left.+\|\operatorname{div} \boldsymbol{v}\|_{L^{p}(\Omega)}+\left\|\boldsymbol{v}_{T}\right\|_{\boldsymbol{W}^{1-1 / p, p}(\Gamma)}\right) .
\end{aligned}
$$

Since $\Omega$ has no holes, we can delete the first term $\|\boldsymbol{u}\|_{\boldsymbol{L}^{p}(\Omega)}$ in the right-hand side of (3.8), that is,

$$
\|\boldsymbol{v}\|_{W^{1, p}(\Omega)} \leq C\left(\|\operatorname{curl} \boldsymbol{v}\|_{L^{p}(\Omega)}+\|\operatorname{div} \boldsymbol{v}\|_{L^{p}(\Omega)}+\left\|\boldsymbol{v}_{T}\right\|_{W^{1-1 / p, p}(\Gamma)}\right) .
$$

In fact, if (3.9) is false, there exists $\left\{\boldsymbol{v}_{n}\right\} \subset \boldsymbol{W}^{1, p}(\Omega)$ such that

$$
1=\left\|\boldsymbol{v}_{n}\right\|_{\boldsymbol{W}^{1, p}(\Omega)}>n\left(\left\|\operatorname{curl} \boldsymbol{v}_{n}\right\|_{\boldsymbol{L}^{p}(\Omega)}+\left\|\operatorname{div} \boldsymbol{v}_{n}\right\|_{L^{p}(\Omega)}+\left\|\boldsymbol{v}_{n, T}\right\|_{\boldsymbol{W}^{1-1 / p, p}(\Gamma)}\right) .
$$

Thus, curl $\boldsymbol{v}_{n} \rightarrow \mathbf{0}$ in $\boldsymbol{L}^{p}(\Omega)$, div $\boldsymbol{v}_{n} \rightarrow 0$ in $L^{p}(\Omega)$ and $\boldsymbol{v}_{n, T} \rightarrow \mathbf{0}$ in $\boldsymbol{W}^{1-1 / p, p}(\Gamma)$. On the other hand, since $\left\|\boldsymbol{v}_{n}\right\|_{\boldsymbol{W}^{1, p}(\Omega)}=1$, passing to a subsequence, we may assume that $\boldsymbol{v}_{n} \rightarrow \boldsymbol{v}$ weakly in $\boldsymbol{W}^{1, p}(\Omega)$ and strongly in $\boldsymbol{L}^{p}(\Omega)$. Hence $\operatorname{curl} \boldsymbol{v}=\mathbf{0}$, div $\boldsymbol{v}=0$ in $\Omega$ and $\boldsymbol{v}_{T}=\mathbf{0}$ on $\Gamma$. Therefore, we have $\boldsymbol{v} \in \mathbb{K}_{N}^{p}(\Omega)$. Since $\Omega$ has no holes, i.e., $\operatorname{dim} \mathbb{K}_{N}^{p}(\Omega)=m=0$, we see that $\boldsymbol{v}=\mathbf{0}$, so $\boldsymbol{v}_{n} \rightarrow \mathbf{0}$ in $\boldsymbol{L}^{p}(\Omega)$. From (3.8), we see that

$$
\begin{aligned}
1= & \left\|\boldsymbol{v}_{n}\right\|_{\boldsymbol{W}^{1, p}(\Omega)} \leq C\left(\left\|\boldsymbol{v}_{n}\right\|_{\boldsymbol{L}^{p}(\Omega)}+\left\|\operatorname{curl} \boldsymbol{v}_{n}\right\|_{\boldsymbol{L}^{p}(\Omega)}\right. \\
& \left.+\left\|\operatorname{div} \boldsymbol{v}_{n}\right\|_{L^{p}(\Omega)}+\left\|\boldsymbol{v}_{n, T}\right\|_{W^{1-1 / p, p}(\Gamma)}\right) \rightarrow 0 \text { as } n \rightarrow \infty .
\end{aligned}
$$

This leads to a contradiction. Hence (3.9) holds. Therefore, we have

$$
\|\boldsymbol{u}\|_{W^{1, p}(\Omega)} \leq C\left(\|\operatorname{curl} \boldsymbol{u}\|_{\boldsymbol{L}^{p}(\Omega)}+\left\|\boldsymbol{u}_{T}^{0}\right\|_{W^{1-1 / p, p}(\Gamma)}\right)
$$

for any $\boldsymbol{u} \in \boldsymbol{W}_{t}^{1, p}\left(\Omega, \operatorname{div} 0, \boldsymbol{u}_{T}^{0}\right)$. If we choose $\varepsilon>0$ small enough, then it follows from (2.4) and (3.9) that there exist positive constants $c, C_{1}$ and $C_{2}$ such that

$$
\begin{aligned}
\mathcal{E}[\boldsymbol{u}]= & \int_{\Omega}\left(\frac{1}{2} S\left(x,|\operatorname{curl} \boldsymbol{u}|^{2}\right)-F(x, \boldsymbol{u})\right) \mathrm{d} x \\
& \geq \frac{\lambda}{p}\|\operatorname{curl} \boldsymbol{u}\|_{\boldsymbol{L}^{p}(\Omega)}^{p}-\varepsilon\|\boldsymbol{u}\|_{\boldsymbol{L}^{p}(\Omega)}^{p}-C_{\varepsilon}|\Omega| \\
& \geq c\|\operatorname{curl} \boldsymbol{u}\|_{L^{p}(\Omega)}^{p}-C_{1}\left\|\boldsymbol{u}_{T}^{0}\right\|_{W^{1-1 / p, p}(\Gamma)}^{p}-C_{2} \\
& \geq-C_{1}\left\|\boldsymbol{u}_{T}^{0}\right\|_{W^{1-1 / p, p}(\Gamma)}^{p}-C_{2}>-\infty .
\end{aligned}
$$

Hence $c_{0}>-\infty$.

Let $\left\{\boldsymbol{u}_{j}\right\} \subset \boldsymbol{W}_{t}^{1, p}\left(\Omega, \operatorname{div} 0, \boldsymbol{u}_{T}^{0}\right)$ be a minimizing sequence of $c_{0}$. Then we have

$$
\mathcal{E}\left[\boldsymbol{u}_{j}\right]=c_{0}+\varepsilon_{j}, \text { where } \varepsilon_{j} \rightarrow 0 \text { as } j \rightarrow \infty .
$$

From (2.4), we have

$$
\frac{\lambda}{p}\left\|\operatorname{curl} \boldsymbol{u}_{j}\right\|_{\boldsymbol{L}^{p}(\Omega)}^{p} \leq \frac{1}{2} \int_{\Omega} S\left(x,\left|\operatorname{curl} \boldsymbol{u}_{j}\right|^{2}\right) \mathrm{d} x=\mathcal{E}\left[\boldsymbol{u}_{j}\right]+\int_{\Omega} F\left(x, \boldsymbol{u}_{j}\right) \mathrm{d} x .
$$

Using (3.7), (3.9) and (3.10), we have

$$
\frac{\lambda}{p}\left\|\operatorname{curl} \boldsymbol{u}_{j}\right\|_{\boldsymbol{L}^{p}(\Omega)}^{p} \leq c_{0}+\varepsilon_{j}+\varepsilon C\left\|\operatorname{curl} \boldsymbol{u}_{j}\right\|_{\boldsymbol{L}^{p}(\Omega)}^{p}+\varepsilon C\left\|\boldsymbol{u}_{T}^{0}\right\|_{W^{1-1 / p, p}(\Gamma)}^{p}+C|\Omega| .
$$


If we choose $\varepsilon>0$ so that $\varepsilon C<\lambda / p$, we can see that $\left\{\boldsymbol{u}_{j}\right\}$ is bounded in $\boldsymbol{W}^{1, p}(\Omega)$. Passing to a subsequence, we may assume that $\boldsymbol{u}_{j} \rightarrow \boldsymbol{u}$ weakly in $\boldsymbol{W}^{1, p}(\Omega)$ and strongly in $\boldsymbol{L}^{p}(\Omega)$. Hence $\operatorname{div} \boldsymbol{u}=0$ in $\Omega$ and $\boldsymbol{u}_{T}=\boldsymbol{u}_{T}^{0}$ on $\Gamma$, so $\boldsymbol{u} \in \boldsymbol{W}_{t}^{1, p}\left(\Omega, \operatorname{div} 0, \boldsymbol{u}_{T}^{0}\right)$. Since $\operatorname{curl} \boldsymbol{u}_{j} \rightarrow \operatorname{curl} \boldsymbol{u}$ weakly in $\boldsymbol{L}^{p}(\Omega)$, it follows from Aramaki [7, Proposition 3.6] that

$$
\int_{\Omega} S\left(x,|\operatorname{curl} \boldsymbol{u}|^{2}\right) \mathrm{d} x \leq \liminf _{j \rightarrow \infty} \int_{\Omega} S\left(x,\left|\operatorname{curl} \boldsymbol{u}_{j}\right|^{2}\right) \mathrm{d} x .
$$

By the hypothesis (3.2),

$$
\begin{aligned}
& \left|\int_{\Omega} F\left(x, \boldsymbol{u}_{j}\right) \mathrm{d} x-\int_{\Omega} F(x, \boldsymbol{u}) \mathrm{d} x\right| \\
& \quad=\left|\int_{\Omega}\left(\boldsymbol{u}_{j}-\boldsymbol{u}\right) \cdot \nabla_{\boldsymbol{u}} F\left(x, \boldsymbol{u}+\theta\left(\boldsymbol{u}_{j}-\boldsymbol{u}\right)\right) \mathrm{d} x\right| \\
& \quad \leq M \int_{\Omega}\left|\boldsymbol{u}_{j}-\boldsymbol{u}\right|\left(1+\left(|\boldsymbol{u}|+\left|\boldsymbol{u}_{j}\right|\right)^{p-1}\right) \mathrm{d} x \\
& \quad \leq M\left\|\boldsymbol{u}_{j}-\boldsymbol{u}\right\|_{\boldsymbol{L}^{p}(\Omega)}\left(\int_{\Omega}\left(1+\left(|\boldsymbol{u}|+\left|\boldsymbol{u}_{j}\right|\right)\right)^{(p-1) p^{\prime}} \mathrm{d} x\right)^{1 / p^{\prime}} \\
& \quad \leq M_{1}\left\|\boldsymbol{u}_{j}-\boldsymbol{u}\right\|_{\boldsymbol{L}^{p}(\Omega)}\left(1+\|\boldsymbol{u}\|_{\boldsymbol{L}^{p}(\Omega)}^{p-1}+\left\|\boldsymbol{u}_{j}\right\|_{\boldsymbol{L}^{p}(\Omega)}^{p-1}\right) .
\end{aligned}
$$

Since $\boldsymbol{u}_{j} \rightarrow \boldsymbol{u}$ strongly in $\boldsymbol{L}^{p}(\Omega)$, we see that $\left\{\left\|\boldsymbol{u}_{j}\right\|_{\boldsymbol{L}^{p}(\Omega)}\right\}$ is bounded. Thus, we have

$$
\lim _{j \rightarrow \infty} \int_{\Omega} F\left(x, \boldsymbol{u}_{j}\right) \mathrm{d} x=\int_{\Omega} F(x, \boldsymbol{u}) \mathrm{d} x .
$$

Summing up (3.11) and (3.12), we see that

$$
\mathcal{E}[\boldsymbol{u}] \leq \liminf _{j \rightarrow \infty} \mathcal{E}\left[\boldsymbol{u}_{j}\right]=c_{0}
$$

Therefore, $\boldsymbol{u}$ is a minimizer of $\mathcal{E}$ on $\boldsymbol{W}_{t}^{1, p}\left(\Omega, \operatorname{div} 0, \boldsymbol{u}_{T}^{0}\right)$.

For any $\boldsymbol{w} \in \boldsymbol{W}_{t 0}^{1, p}(\Omega, \operatorname{div} 0)$, we see that $\boldsymbol{u}+\tau \boldsymbol{w} \in \boldsymbol{W}_{t}^{1, p}\left(\Omega, \operatorname{div} 0, \boldsymbol{u}_{T}^{0}\right)$ for any $\tau \in \mathbb{R}$. Since $\boldsymbol{u}$ is a minimizer of $\mathcal{E}$, we have

$$
\begin{aligned}
0 & =\left.\frac{\mathrm{d}}{\mathrm{d} \tau}\right|_{\tau=0} \mathcal{E}[\boldsymbol{u}+\tau \boldsymbol{w}] \\
& =\int_{\Omega} S_{t}\left(x,|\operatorname{curl} \boldsymbol{u}|^{2}\right) \operatorname{curl} \boldsymbol{u} \cdot \operatorname{curl} \boldsymbol{w} \mathrm{d} x-\int_{\Omega} \boldsymbol{f}(x, \boldsymbol{u}) \cdot \boldsymbol{w} \mathrm{d} x
\end{aligned}
$$

for any $\boldsymbol{w} \in \boldsymbol{W}_{t 0}^{1, p}(\Omega, \operatorname{div} 0)$. By a version of the de Rham lemma (Theorem 2.2), there exists $\pi \in L^{p^{\prime},-1 / p^{\prime}}(\Omega)$ such that

$$
\operatorname{curl}\left[S_{t}\left(x,|\operatorname{curl} \boldsymbol{u}|^{2}\right) \operatorname{curl} \boldsymbol{u}\right]-\boldsymbol{f}(x, \boldsymbol{u})=\nabla \pi \text { in } \boldsymbol{W}_{t 0}^{1, p}(\Omega)^{\prime}
$$

and $\pi=0$ on $\Gamma$ in the sense of trace. Therefore, $(\boldsymbol{u}, \pi)$ is a weak solution of (3.1).

Furthermore, assume that $\boldsymbol{f}$ satisfies (3.6). Let $\left(\boldsymbol{u}_{1}, \pi_{1}\right)$ and $\left(\boldsymbol{u}_{2}, \pi_{2}\right)$ be two weak solutions of (3.1). If we take $\boldsymbol{u}_{1}-\boldsymbol{u}_{2} \in \boldsymbol{W}_{t 0}^{1, p}(\Omega)$ as a test function of (3.5), we have

$$
\int_{\Omega} S_{t}\left(x,\left|\operatorname{curl} \boldsymbol{u}_{1}\right|^{2}\right) \operatorname{curl} \boldsymbol{u}_{1} \cdot \operatorname{curl}\left(\boldsymbol{u}_{1}-\boldsymbol{u}_{2}\right) \mathrm{d} x=\int_{\Omega} \boldsymbol{f}\left(x, \boldsymbol{u}_{1}\right) \cdot\left(\boldsymbol{u}_{1}-\boldsymbol{u}_{2}\right) \mathrm{d} x
$$

and

$$
\int_{\Omega} S_{t}\left(x,\left|\operatorname{curl} \boldsymbol{u}_{2}\right|^{2}\right) \operatorname{curl} \boldsymbol{u}_{2} \cdot \operatorname{curl}\left(\boldsymbol{u}_{1}-\boldsymbol{u}_{2}\right) \mathrm{d} x=\int_{\Omega} \boldsymbol{f}\left(x, \boldsymbol{u}_{2}\right) \cdot\left(\boldsymbol{u}_{1}-\boldsymbol{u}_{2}\right) \mathrm{d} x .
$$


Here we used the fact that since $\operatorname{div} \boldsymbol{u}_{i}=0$ in $\Omega$ and $\pi_{j}=0$ on $\Gamma$, by the integration by parts we have

$$
\int_{\Omega} \nabla \pi_{j} \cdot \boldsymbol{u}_{i} \mathrm{~d} x=0 \text { for } i, j=1,2 .
$$

Thus we have

$$
\begin{aligned}
& \int_{\Omega}\left(S_{t}\left(x,\left|\operatorname{curl} \boldsymbol{u}_{1}\right|^{2}\right) \operatorname{curl} \boldsymbol{u}_{1}-S_{t}\left(x,\left|\operatorname{curl} \boldsymbol{u}_{2}\right|^{2}\right) \operatorname{curl} \boldsymbol{u}_{2}\right) \cdot \operatorname{curl}\left(\boldsymbol{u}_{1}-\boldsymbol{u}_{2}\right) \mathrm{d} x \\
& \quad=\int_{\Omega}\left(\boldsymbol{f}\left(x, \boldsymbol{u}_{1}\right)-\boldsymbol{f}\left(x, \boldsymbol{u}_{2}\right) \cdot\left(\boldsymbol{u}_{1}-\boldsymbol{u}_{2}\right) \mathrm{d} x\right.
\end{aligned}
$$

If we use the monotonicity of $S_{t}$ (cf. [4, Lemma 3.6]):

$$
\begin{aligned}
& \left(S_{t}\left(x,|\boldsymbol{a}|^{2}\right) \boldsymbol{a}-S_{t}\left(x,|\boldsymbol{b}|^{2}\right) \boldsymbol{b}\right) \cdot(\boldsymbol{a}-\boldsymbol{b}) \\
& \geq\left\{\begin{array}{cl}
c|\boldsymbol{a}-\boldsymbol{b}|^{p} & \text { if } p>2, \\
c(|\boldsymbol{a}|+|\boldsymbol{b}|)^{p-2}|\boldsymbol{a}-\boldsymbol{b}|^{2} & \text { if } 1<p \leq 2,
\end{array}\right.
\end{aligned}
$$

with some positive constant $c$, then from (3.6) we have

$$
c \int_{\Omega}\left|\operatorname{curl}\left(\boldsymbol{u}_{1}-\boldsymbol{u}_{2}\right)\right|^{p} \mathrm{~d} x \leq 0 \text { if } p>2
$$

and

$$
c \int_{\Omega}\left(\left|\operatorname{curl} \boldsymbol{u}_{1}\right|+\left|\operatorname{curl} \boldsymbol{u}_{2}\right|\right)^{p-2}\left|\operatorname{curl}\left(\boldsymbol{u}_{1}-\boldsymbol{u}_{2}\right)\right|^{2} \mathrm{~d} x \leq 0 \text { if } 1<p \leq 2 .
$$

These imply curl $\left(\boldsymbol{u}_{1}-\boldsymbol{u}_{2}\right)=\mathbf{0}, \operatorname{div}\left(\boldsymbol{u}_{1}-\boldsymbol{u}_{2}\right)=0$ in $\Omega$ and $\left(\boldsymbol{u}_{1}-\boldsymbol{u}_{2}\right)_{T}=\mathbf{0}$ on $\Gamma$. Hence $\boldsymbol{u}_{1}-\boldsymbol{u}_{2} \in \mathbb{K}_{N}^{p}(\Omega)$. Since $\Omega$ has no holes, we see that $\boldsymbol{u}_{1}=\boldsymbol{u}_{2}$ in $\Omega$. Taking divergence of the first equation of $(3.1), \pi_{i}(i=1,2)$ satisfies

$$
\begin{cases}\Delta \pi_{i}=-\operatorname{div} \boldsymbol{f}\left(x, \boldsymbol{u}_{1}(x)\right) & \text { in } \Omega \\ \pi_{i}=0 & \text { on } \Gamma\end{cases}
$$

for $i=1,2$. From the uniqueness of Dirichlet problem for the Poisson equation, we get $\pi_{1}=\pi_{2}$. This completes the proof.

\section{Regularity of weak solutions}

In this section, we consider the regularity of weak solutions to the following system:

$$
\begin{cases}\operatorname{curl}\left[S_{t}\left(x,|\operatorname{curl} \boldsymbol{u}|^{2}\right) \operatorname{curl} \boldsymbol{u}\right]=\boldsymbol{J}(x)+\nabla \pi & \text { in } \Omega, \\ \operatorname{div} \boldsymbol{u}=0 & \text { in } \Omega, \\ \boldsymbol{u}_{T}=\mathbf{0} & \text { on } \Gamma, \\ \pi=0 & \text { on } \Gamma,\end{cases}
$$

where $\boldsymbol{J} \in \boldsymbol{C}^{\alpha}(\bar{\Omega})$ is a given function.

We have the following theorem.

Theorem 4.1 Assume that $\Omega$ is a bounded domain in $\mathbb{R}^{3}$ with a $C^{2}$ boundary $\Gamma$ and $\Omega$ has no holes. Let $\boldsymbol{J} \in \boldsymbol{C}^{\alpha}(\bar{\Omega})(0<\alpha<1)$. Then (4.1) has a unique weak solution $(\boldsymbol{u}, \pi) \in \boldsymbol{W}_{t 0}^{1, p}(\Omega, \operatorname{div} 0) \times L^{p^{\prime},-1 / p^{\prime}}(\Omega)$. Moreover, $\pi \in C^{1, \alpha}(\bar{\Omega})$ and $\boldsymbol{u} \in \boldsymbol{C}^{1, \beta}(\bar{\Omega})$ for some $\beta \in(0,1)$ and

$$
\|\pi\|_{C^{1, \alpha}(\bar{\Omega})} \leq C_{1}\|\boldsymbol{J}\|_{C^{\alpha}(\bar{\Omega})}
$$

and

$$
\|\boldsymbol{u}\|_{\boldsymbol{C}^{1, \beta}(\bar{\Omega})} \leq C_{2},
$$

where $C_{1}$ depends only on $\Omega$ and $C_{2}$ depends on $p, \alpha$ and $\|\boldsymbol{J}\|_{C^{\alpha}(\bar{\Omega})}$. 
Proof If we put $F(x, z)=\boldsymbol{J}(x) \cdot \boldsymbol{z}$, then $F$ clearly satisfies (3.4), (3.5) and (3.6). Hence it follows from Theorem 3.2 that the weak solution of (4.1) uniquely exists. Let $(\boldsymbol{u}, \pi)$ be the weak solution of (4.1). Then $\pi$ satisfies

$$
\begin{cases}-\Delta \pi=\operatorname{div} \boldsymbol{J} & \text { in } \Omega \\ \pi=0 & \text { on } \Gamma .\end{cases}
$$

Since $\boldsymbol{J} \in \boldsymbol{C}^{\alpha}(\bar{\Omega})$, it follows from Gilbarg and Trudinger [11, Theorem 8.34, 8.33] that $\pi \in C^{1, \alpha}(\bar{\Omega})$, and there exists a constant $C$ depending only on $\Omega$ such that

$$
\|\pi\|_{C^{1, \alpha}(\bar{\Omega})} \leq C\left(\|\pi\|_{C^{0}(\bar{\Omega})}+\|\boldsymbol{J}\|_{C^{\alpha}(\bar{\Omega})}\right) .
$$

However, we can delete the first term of the right-hand side of (4.3), i.e., the following estimate holds.

$$
\|\pi\|_{C^{1, \alpha}(\bar{\Omega})} \leq C\|\boldsymbol{J}\|_{C^{\alpha}(\bar{\Omega})} .
$$

In fact, suppose that (4.4) is false. Then there exist $\left\{\pi_{k}\right\} \subset C^{1, \alpha}(\bar{\Omega})$ and $\left\{\boldsymbol{J}_{k}\right\} \subset \boldsymbol{C}^{\alpha}(\bar{\Omega})$ such that $\pi_{k}$ is a weak solution of

$$
\begin{cases}-\Delta \pi_{k}=\operatorname{div} \boldsymbol{J}_{k} & \text { in } \Omega \\ \pi_{k}=0 & \text { on } \Gamma\end{cases}
$$

and

$$
1=\left\|\pi_{k}\right\|_{C^{1, \alpha}(\bar{\Omega})}>k\left\|J_{k}\right\|_{C^{\alpha}(\bar{\Omega})}
$$

Then $\boldsymbol{J}_{k} \rightarrow \mathbf{0}$ in $\boldsymbol{C}^{\alpha}(\bar{\Omega})$ and

$$
\int_{\Omega} \nabla \pi_{k} \cdot \nabla \varphi \mathrm{d} x=-\int_{\Omega} \boldsymbol{J}_{k} \cdot \nabla \varphi \mathrm{d} x \text { for all } \varphi \in W_{0}^{1, p^{\prime}}(\Omega) .
$$

Since $\left\|\pi_{k}\right\|_{C^{1, \alpha}(\bar{\Omega})}=1$, there exists a subsequence $\left\{\pi_{k_{l}}\right\}$ such that $\pi_{k_{l}} \rightarrow \pi$ in $C^{1, \gamma}(\bar{\Omega})$ as $l \rightarrow \infty$ for any $0<\gamma<\alpha$. In particular, $\pi_{k_{l}} \rightarrow \pi$ in $W_{0}^{1, p}(\Omega)$. Letting $l \rightarrow \infty$ in (4.5) with $k=k_{l}$, we have

$$
\int_{\Omega} \nabla \pi \cdot \nabla \varphi \mathrm{d} x=0 \text { for all } \varphi \in W_{0}^{1, p^{\prime}}(\Omega) .
$$

That is, $\pi$ is a weak solution of

$$
\begin{cases}-\Delta \pi=0 & \text { in } \Omega \\ \pi=0 & \text { on } \Gamma\end{cases}
$$

By the uniqueness of the Dirichlet problem for the Poisson equation, we have $\pi=0$, so $\pi_{k_{l}} \rightarrow 0$ in $C^{0}(\bar{\Omega})$. Hence by (4.3), we have

$$
1=\left\|\pi_{k_{l}}\right\|_{C^{1, \alpha}(\bar{\Omega})} \leq C\left(\left\|\pi_{k_{l}}\right\|_{C^{0}(\bar{\Omega})}+\left\|\boldsymbol{J}_{k}\right\|_{C^{\alpha}(\bar{\Omega})}\right) \rightarrow 0
$$

as $l \rightarrow \infty$. This leads to a contradiction. Therefore, (4.4) holds.

Since $\boldsymbol{J}+\nabla \pi \in \boldsymbol{C}^{\alpha}(\bar{\Omega})$, div $(\boldsymbol{J}+\nabla \pi)=0$ in $\Omega$ and $\Omega$ has no holes, it follows from [4, Theorem 2.2] that there exists $\beta \in(0,1)$ such that $\boldsymbol{u} \in \boldsymbol{C}^{1, \beta}(\bar{\Omega})$, and

$$
\|\boldsymbol{u}\|_{\boldsymbol{C}^{1, \beta}(\bar{\Omega})} \leq C,
$$

where $C$ depends on $p, \alpha$ and $\|\boldsymbol{J}\|_{\boldsymbol{C}^{\alpha}(\bar{\Omega})}$.

Open Access This article is distributed under the terms of the Creative Commons Attribution 4.0 International License (http:// creativecommons.org/licenses/by/4.0/), which permits unrestricted use, distribution, and reproduction in any medium, provided you give appropriate credit to the original author(s) and the source, provide a link to the Creative Commons license, and indicate if changes were made. 


\section{References}

1. Amrouche, C.; Seloula, N.H.: $L^{p}$-theory for vector potentials and Sobolev's inequality for vector fields. Application to the Stokes equations with pressure boundary conditions. Math. Models Methods Appl. Sci. 23, 37-92 (2013)

2. Amrouche, C.; Seloula, N.H.: $L^{p}$-theory for vector potentials and Sobolev's inequality for vector fields. C. R. Acad. Sci. Paris Ser. 1 349, 529-534 (2011)

3. Aramaki, J.: Regularity of weak solutions for degenerate quasilinear elliptic equations involving operator curl. J. Math. Anal. Appl. 426, 872-892 (2015)

4. Aramaki, J.: Existence and regularity of a weak solution to a class of systems in a multi-connected domain (submitted)

5. Aramaki, J.: A version of the de Rham lemma (submitted)

6. Aramaki, J.: Existence and regularity for the Neumann problem to the Poisson equation and an application to the MaxwellStokes type equation. Comm. Math. Anal. 21, 54-66 (2018)

7. Aramaki, J.: Variational problem involving operator curl associated with p-curl system. Turk. J. Math. 42, 949-966 (2018)

8. Boyer, F.; Fabrie, P.: Mathematical Tools for the Study of the Incompressible Navier-Stokes Equations and Related Models. Springer Science+Business Media, Yew York (2013)

9. Chapman, J.: A hierarchy of models for type-II superconductors. SIAM Rev. 42, 555-598 (2000)

10. Dautray, R.; Lions, J.L.: Mathematical Analysis and Numerical Method for Science and Technology, vol. 3. Springer, New York (1990)

11. Gilbarg, D.; Trudinger, N.S.: Elliptic Partial Differential Equations of Second Order. Springer, Berlin (1998)

12. Girault, V.; Raviart, P.A.: Finite Element Methods for Navier-Stokes Equations. Springer, Berlin (1979)

13. Pan, X.-B.: Existence and regularity of solutions to quasilinear systems of Maxwell and Maxwell-Stokes type. Calc. Var. Part. Differ. Equ. 55, 1-43 (2016)

14. Prigozhin, L.: On the Bean critical-state model in superconductivity. Eur. J. Appl. Math. 7, 237-247 (1996)

15. Yin, H.-M.: On a $p$-Laplacian type of evolution system and applications to the Bean model in the type-II superconductivity theory. Q. Appl. Math. LIX, 47-66 (2001)

16. Yin, H.-M.: Regularity of weak solution to a $p$ - curl-system. Differ. Integral Equ. 19(4), 361-368 (2006)

17. Yin, H.M.; Li, B.Q.; Zou, J.: A degenerate evolution system modeling Bean's critical state type-II superconductors. Discret. Contin. Dyn. Syst. 8, 781-794 (2002)

Publisher's Note Springer Nature remains neutral with regard to jurisdictional claims in published maps and institutional affiliations. 